05

\title{
Динамика магнитного момента ограниченных дипольных решеток в переменном поле
}

\author{
(С) А.М. Шутый, Д.И. Семенцов \\ Ульяновский государственный университет, \\ Ульяновск, Россия \\ E-mail: shuty@mail.ru \\ (Поступила в Редакцию 14 июня 2016 г.)
}

Исследованы динамические режимы магнитного момента квадратных дипольных решеток $2 \times 2,3 \times 3$ и $4 \times 4$ в переменном магнитном поле с линейной и круговой поляризацией при ортогональном ему статическом магнитном поле. Показана возможность реализации регулярной и хаотической прецессионной динамики магнитного момента решеток. Выявлено смещение основного магнитного резонанса, обусловленное диполь-дипольным взаимодействием, а также дополнительные резонансы. Обнаружены квазипериодические режимы и состояния бистабильности с метастабильным хаотическим аттрактором.

Работа проведена в рамках выполнения задания Министерства образования и науки РФ (проекты № 3.175.2014K, 14.Z50.31.0015).

DOI: 10.21883/FTT.2017.01.43953.244

\section{1. Введение}

В последние годы ведется активное изучение создаваемых с помощью нанотехнологий магнитных сверхструктур и ансамблей магнитных частиц [1-7]. Среди таких структур особый интерес представляют состоящие из однодоменных нанодиполей одномерные и двумерные структуры, в частности магнитоупорядоченные квадратные решетки. Подобные структуры могут быть сформированы, например, на основе наночастиц ферромагнитных металлов [8]. Основной вклад во взаимодействие магнитных моментов в таких решетках вносит дипольдипольное взаимодействие $[8,9]$. В работах [10-13] рассмотрены равновесные состояния в линейных цепочках и квадратных решетках магнитных моментов нанодиполей и динамические режимы, возникающие в процессе их перемагничивания во внешнем статическом магнитном поле. Дискретность структур приводит к существенным отличиям статических и динамических свойств подобных решеток от свойств макроскопических монодоменных объектов. К таким отличиям, в частности, могут быть отнесены бистабильные состояния систем, обусловленные наличием разных ориентационных конфигураций, различающихся суммарным магнитным моментом систем, а также осуществление управляемых переходов между данными конфигурациями и возникновение динамических колебательных режимов магнитного момента системы при их перемагничивании.

Следует ожидать различий и в динамическом поведении таких структур в переменных магнитных полях. В настоящей работе на примере плоских квадратных решеток типа $n \times n$ с $n=2,3,4$ исследуется резонансная динамика дипольных систем. С помощью бифуркационных диаграмм (БД) выявляются условия реализации периодических, квазипериодических и хаотических динамических режимов при различных значениях статического подмагничивающего поля, ориентированного перпендикулярно плоскости решетки, и разных поляризациях переменного поля.

\section{2. Исходные уравнения}

Рассмотрим систему магнитных нанодиполей с одинаковыми магнитными моментами $\mathbf{m}_{i}$. Считаем, что они связаны диполь-дипольным взаимодействием. В этом случае уравнения Ландау-Лифшица для каждого из моментов решетки записываются следующим образом [14]:

$$
\frac{\partial \mathbf{m}_{i}}{\partial t}=-\gamma \mathbf{m}_{i} \times \mathbf{H}_{i}^{\mathrm{eff}}-\frac{\alpha_{i}}{m_{i}} \mathbf{m}_{i} \times \frac{\partial \mathbf{m}_{i}}{\partial t},
$$

где $\alpha_{i}$ - параметр диссипации, $\gamma-$ гиромагнитное отношение. Эффективное поле, создаваемое в месте расположения $i$-го диполя остальными диполями и внешним магнитным полем $\mathbf{H}$, определяется выражением:

$$
\mathbf{H}_{i}^{\mathrm{eff}}=\mathbf{H}+\sum_{n \neq i}\left[\frac{3\left(\mathbf{m}_{n} \mathbf{r}_{i n}\right) \mathbf{r}_{i n}-\mathbf{m}_{n} r_{i n}^{2}}{r_{i n}^{5}}\right],
$$

где $\mathbf{r}_{i n}$ и $r_{i n}-$ радиус-вектор и расстояние между $i$-м и $n$-м диполями. Поскольку магнитные моменты в системе являются идентичными, то $\left|\mathbf{m}_{i}\right|=m, \alpha_{i}=\alpha$. Далее перейдем к безразмерным параметрам: $\mathbf{e}_{i n}=\mathbf{r}_{i n} / r_{i n}$, $\tau=\left(m \gamma / a^{3}\right) t$, где $a-$ расстояние между ближайшими магнитными моментами, $l_{i n}=r_{i n} / a, \boldsymbol{\mu}_{i}=\mathbf{m}_{i} / m$, внешнее поле в этом случае равно $\mathbf{h}=\mathbf{H} a^{3} / m$ (для величин $m \approx 3 \mu_{\mathrm{B}}, a \approx 5 \mathrm{~nm} \mathrm{и} \gamma=1.76 \cdot 10^{7} \mathrm{Oe} \cdot \mathrm{s}^{-1}$ значения времени $t \approx 2.55 \cdot 10^{-7} \tau$ [s] и поля $\left.H \approx 0.22 h[\mathrm{Oe}]\right)$. В безразмерных параметрах уравнения (1) примут вид

$$
\frac{\partial \boldsymbol{\mu}_{i}}{\partial \tau}=-\boldsymbol{\mu}_{i} \times \mathbf{h}_{i}^{\mathrm{eff}}-\alpha \boldsymbol{\mu}_{i} \times \frac{\partial \boldsymbol{\mu}_{i}}{\partial \tau},
$$


где

$$
\mathbf{h}_{i}^{\mathrm{eff}}=\mathbf{h}+\sum_{n \neq i}\left[\frac{3\left(\boldsymbol{\mu}_{n} \mathbf{e}_{i n}\right) \mathbf{e}_{i n}-\boldsymbol{\mu}_{n}}{l_{i n}^{3}}\right] .
$$

При дальнейшем анализе векторное уравнение (3) представляется тремя скалярными уравнениями. Так, для $x$-компонент $\partial \boldsymbol{\mu}_{i} / \partial \tau$ получаем

$$
\begin{aligned}
\left(1+\alpha^{2}\right) \frac{\partial \mu_{i x}}{\partial \tau} & =\left(\mu_{i z}+\alpha \mu_{i x} \mu_{i y}\right) h_{i y}^{\mathrm{eff}} \\
& -\left(\mu_{i y}-\alpha \mu_{i z} \mu_{i x}\right) h_{i z}^{\mathrm{eff}}-\alpha\left(1-\mu_{i x}^{2}\right) h_{i x}^{\mathrm{eff}} .
\end{aligned}
$$

Уравнения для остальных компонент величины $\partial \boldsymbol{\mu}_{i} / \partial \tau$ имеют аналогичный вид и могут быть получены циклической перестановкой составляющих. Система координат выбрана таким образом, что ось $O X$ перпендикулярна плоскости решетки, а две другие оси параллельны сторонам решетки.

\section{3. Равновесные конфигурации}

Равновесные конфигурации и динамические режимы определяются на основе численного анализа, который проводится с помощью метода Рунге-Кутта четвертого порядка с учетом связи всех элементов ансамбля друг с другом. При отсутствии внешних полей равновесные конфигурации находятся заданием произвольных начальных состояний магнитных моментов всех дипо-

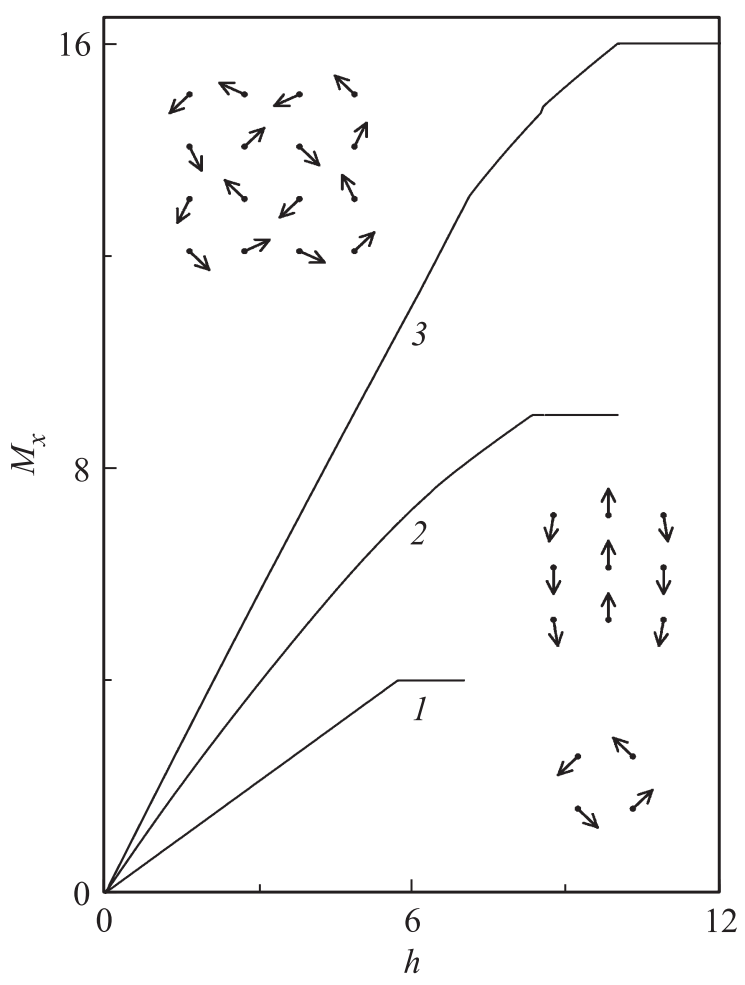

Рис. 1. Зависимость нормальной компоненты суммарного магнитного момента систем $2 \times 2(1), 3 \times 3$ (2) и $4 \times 4$ (3) от величины статического поля, приложенного перпендикулярно плоскости решеток, и конфигурации в плоскости $y z$ решеток в отсутствие подмагничивающего поля. лей, после чего система приходит к стационарному состоянию согласно приведенным уравнениям движения. При действии на решетку в заданном направлении внешнего статического поля система перемагничивается, в результате чего устанавливается новая конфигурация магнитных моментов.

Далее рассмотрим плоские квадратные дипольные решетки типа $n \times n$ при действии на них подмагничивающего поля, направленного вдоль нормали к плоскости решетки. На рис. 1 приведена зависимость $x$-компоненты суммарного магнитного момента $\mathbf{M}=\sum \boldsymbol{\mu}_{i}$ от величины статического поля для решеток с $n=2,3,4$ (кривые 1-3). Видно, что для решетки $2 \times 2$ данная зависимость до поля насыщения, начиная с которого все диполи ориентированы по полю, является практически линейной. Для решеток с $n=3,4$ при приближении к насыщению скорость изменения величины $\mathbf{M}_{x}$ уменьшается, и линейный характер зависимости $M_{x}(h)$ нарушается. С увеличением числа диполей в решетке величина поля насыщения возрастает, что объясняется понижением магнитостатической энергии системы, обусловленной диполь-дипольным взаимодействием. На рисунке также приведены исходные конфигурации (в плоскости $y z$ ) магнитных моментов отдельных диполей решеток, peaлизуемые в отсутствие внешнего поля. Суммарный магнитный момент систем с $n=2,4$ в исходном состоянии равен нулю, система же с $n=3$ имеет плоскостную компоненту магнитного момента.

\section{4. Динамические режимы суммарного магнитного момента}

Рассмотрим колебания магнитного момента дипольных решеток при воздействии статического и переменного магнитных полей $\mathbf{H}=\mathbf{H}_{c}+\mathbf{H}_{p}$. Подмагничивающее поле направим перпендикулярно плоскости решетки, т.е. $\mathbf{h}_{c}=h_{c} \mathbf{e}_{x}$, а переменное поле зададим линейно поляризованным в плоскости решетки $H_{p}=H_{0} \sin (\omega t)$, в приведенных величинах оно записывается в виде $\mathbf{h}_{p}=h_{0} \sin (\Omega \tau) \mathbf{e}_{y}$, где $\Omega=\left(a^{3} / m \gamma\right) \omega$. Анализ будем проводить с помощью БД для $y$-компоненты магнитного момента решетки на плоскости $\left(M_{y m}, \Omega\right)[15,16]$. На данных диаграммах каждому значению частоты переменного поля $\Omega$ соответствуют экстремальные значения суммарной $y$-компоненты магнитного момента $M_{y} \max$ и $M_{y} \min$. Если на диаграмме одному значению $\Omega$ отвечают две или большее конечное число точек, то реализуются регулярные прецессионные режимы; множеству близкорасположенных точек отвечает хаотическая или квазипериодическая динамика.

На рис. 2 приведены БД для $y$-компонент изолированного диполя, а на рис. 3 - для $y$-компонент суммарного магнитного момента дипольных систем с $n=2,3,4$ (диаграммы 1-3). Зависимости получены при значениях подмагничивающего поля $h_{c}=100,50,10$, $5(a-d)$ и амплитуде переменного поля $h_{0}=1$. Параметр диссипации здесь и далее принят равным $\alpha=0.01$. 


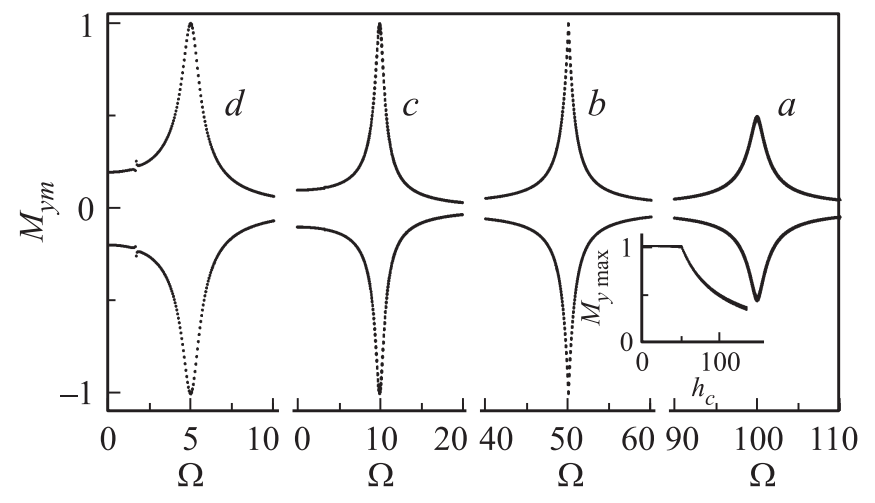

Pис. 2. Диаграммы зависимости экстремумов $y$-компоненты магнитного момента изолированного диполя от частоты переменного поля $\Omega$ при его амплитуде $h_{0}=1$ и величине подмагничивающего поля $h_{c}=100(a), 50(b), 10(c), 5(d)$; параметр диссипации $\alpha=0.01$. На вставке - зависимость от $h_{c}$ амплитуды прецессии магнитного момента диполя на частоте $\Omega_{0}=h_{c}$ при $h_{0}=1$.
Резонансная частота изолированных магнитных моментов в статическом магнитном поле $\omega_{0}=\gamma H_{c}$ в безразмерном виде дается выражением

$$
\Omega_{0}=\frac{a^{3} \omega_{0}}{m \gamma}=h_{c}
$$

что подтверждается результатами численного анализа. На вставке к рис. 2 приведена зависимость от величины подмагничивающего поля $h_{c}$ амплитуды прецессии магнитного момента диполя на резонансной частоте $\Omega_{0}=h_{c}$, из которой следует, что при $h_{c} \leq 50$ достигается амплитуда $M_{y \max } \approx 1$, а при увеличении подмагничивающего поля резонансная амплитуда падает.

Из приведенных на рис. 2 и 3 зависимостей следует, что влияние диполь-дипольного взаимодействия смещает данную частоту в сторону меньших значений. При этом увеличивается ширина резонансной кривой, и резонанс, как правило, имеет нелинейный характер:
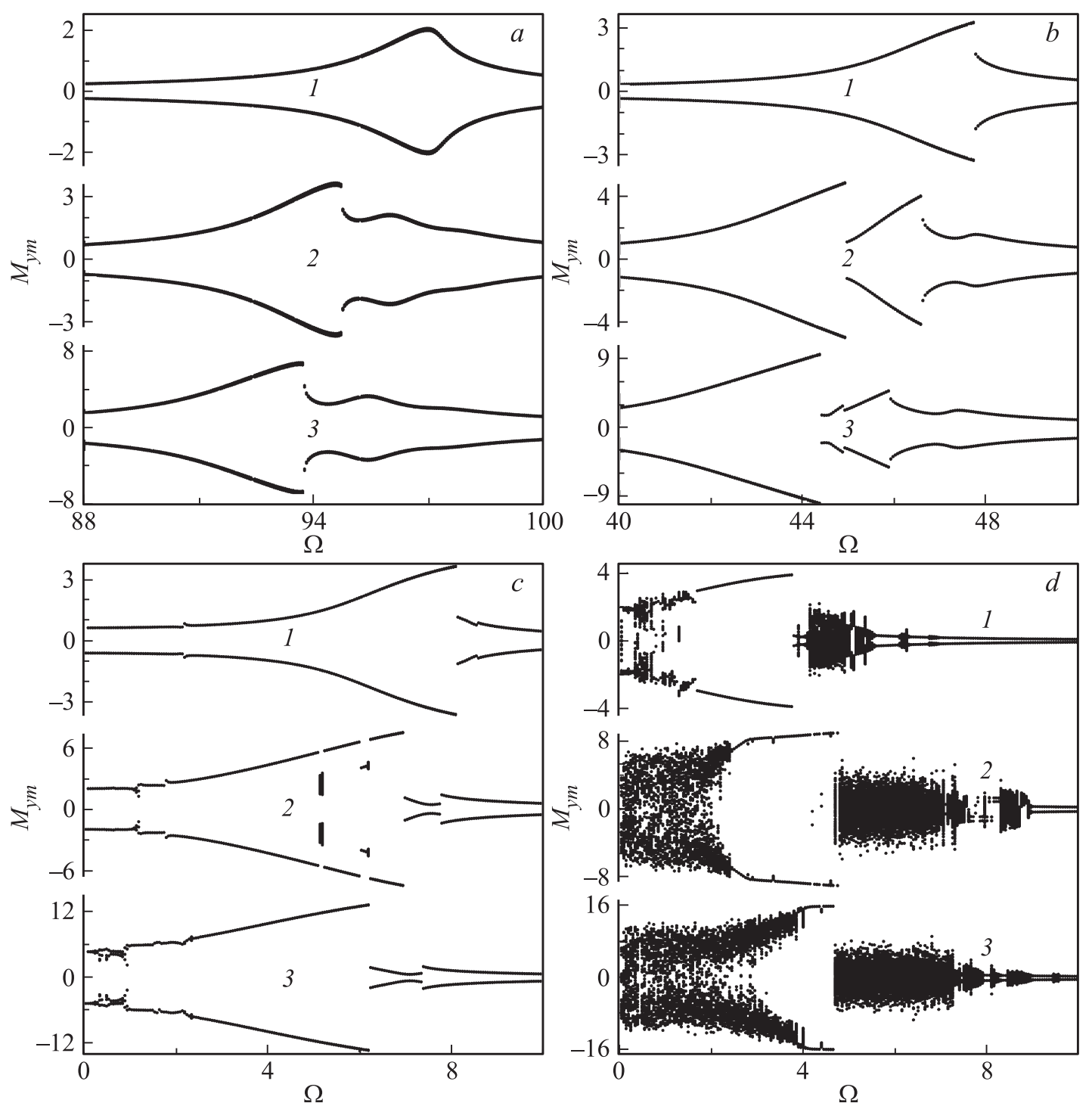

Рис. 3. Диаграммы зависимости экстремумов $y$-компоненты суммарного магнитного момента решеток $2 \times 2(1), 3 \times 3(2)$ и $4 \times 4(3)$ от частоты переменного поля $\Omega$ при $h_{0}=1$ и $h_{c}=100(a), 50(b), 10(c)$ и $5(d)$. 

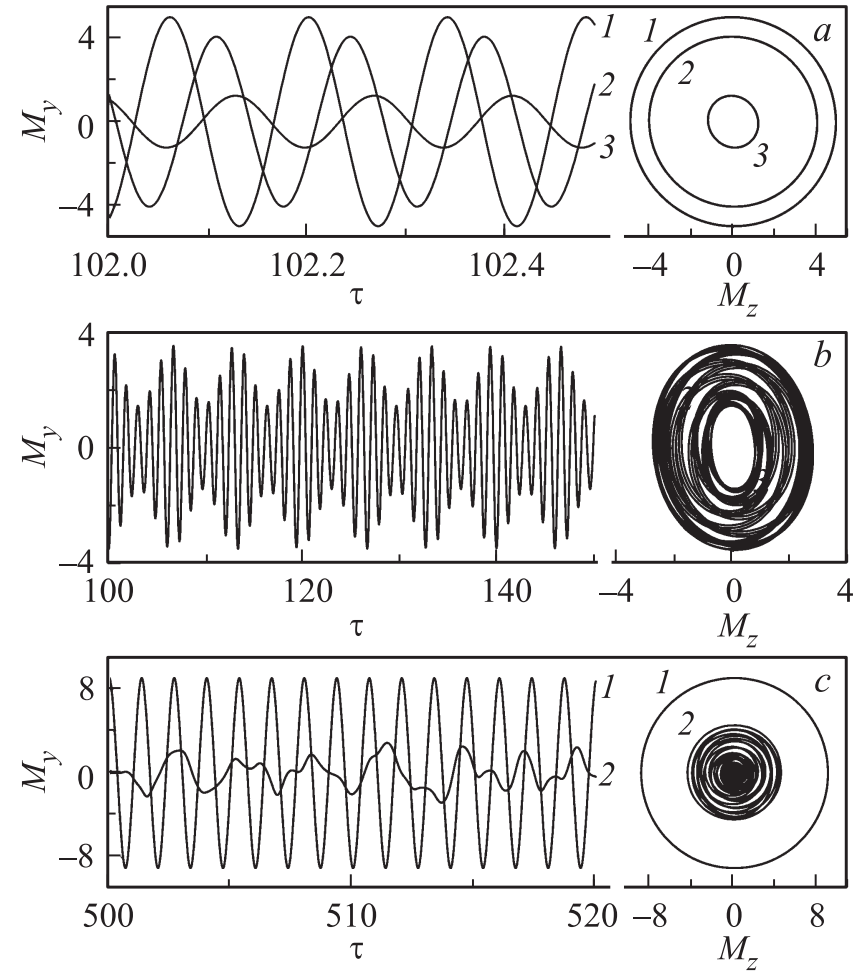

Рис. 4. Зависимость от времени $y$-компоненты магнитного момента системы $3 \times 3$ и проекции на плоскости решетки траектории прецессионных режимов при подмагничивающем поле $h_{c}=50(a), 10(b), 5(c)$ и амплитуде переменного поля $h_{0}=1$ на частотах $\Omega=45.3(1), 46.9(2), 47$ (3) (a), 4.7 (b) и $5.2(c)$.

с увеличением частоты переменного поля наблюдается медленный рост амплитуды прецессии до резонансных значений и резкое ее падение. В случае систем с $n=3,4$ при $100<h_{c}<10$ имеет место явно выраженный второй резонанс, причем в наибольшей степени он проявляется у решеток $3 \times 3$. При относительно слабых подмагничивающих полях $h_{c} \leq 5$ преобладают частотные интервалы, отвечающие хаотической динамике магнитного момента системы. Интервалы же, отвечающие регулярным колебаниям, сокращаются с увеличением числа диполей в решетке.

На рис. 4 для решеток $3 \times 3$ приведена зависимость от времени $y$-компоненты суммарного магнитного момента и проекции на плоскость $y z$ траекторий колебательных режимов при подмагничивающем поле $h_{c}=50$, $10,5(a-c)$ и амплитуде переменного поля $h_{0}=1$ на частотах $\Omega=45.3,46.9,47(a), 4.7(b), 5.2(c)$. Частоты на рис. 4, $a$ близки к двум резонансным значениям и промежуточному значению, отвечающему минимальной амплитуде прецессии. Видно, что во всех случаях колебания близки к гармоническим, переменными являются плоскостные компоненты суммарного магнитного момента, а компонента, перпендикулярная плоскости системы, близка к константе. Рис. $4, b$ отвечает квазипериодическому режиму, когда при $\tau \rightarrow \infty$ траектория
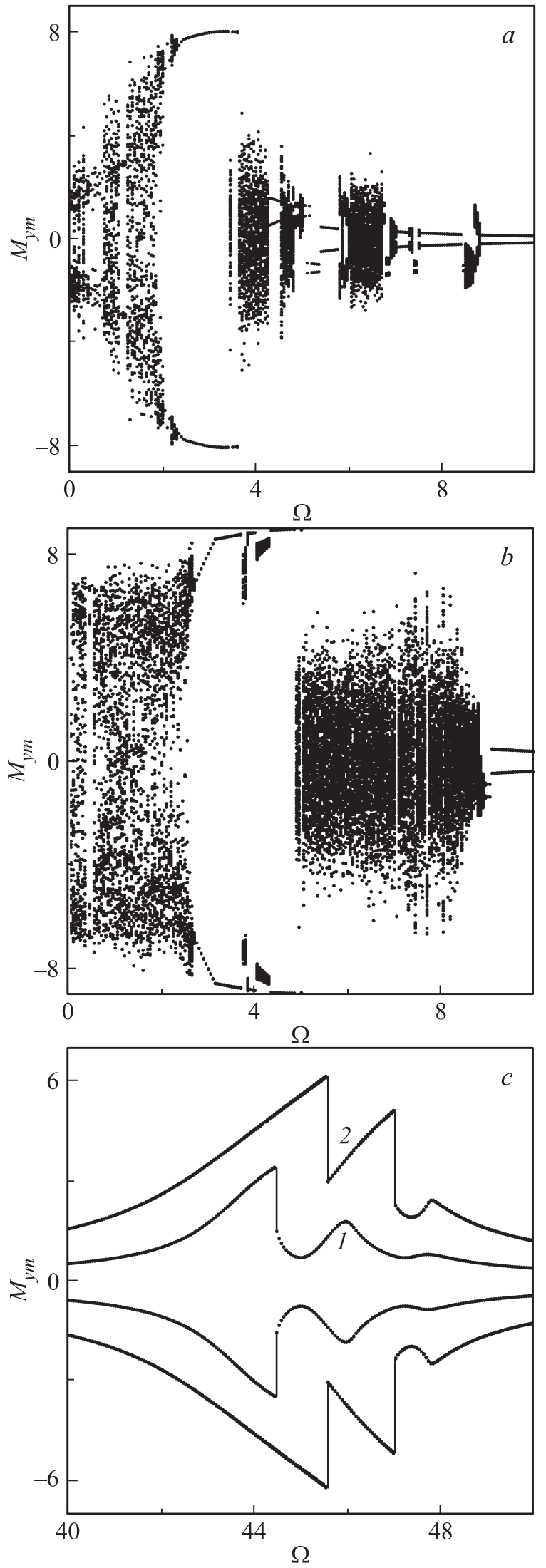

Рис. 5. Диаграммы зависимости динамических режимов магнитного момента системы $3 \times 3$ от частоты переменного поля при его амплитуде $h_{0}=0.5(a ; c-$ кривая 1$)$ и $1.5(b ; c-$ кривая 2) и подмагничивающем поле $h_{c}(a, b)$ и $50(c)$. 

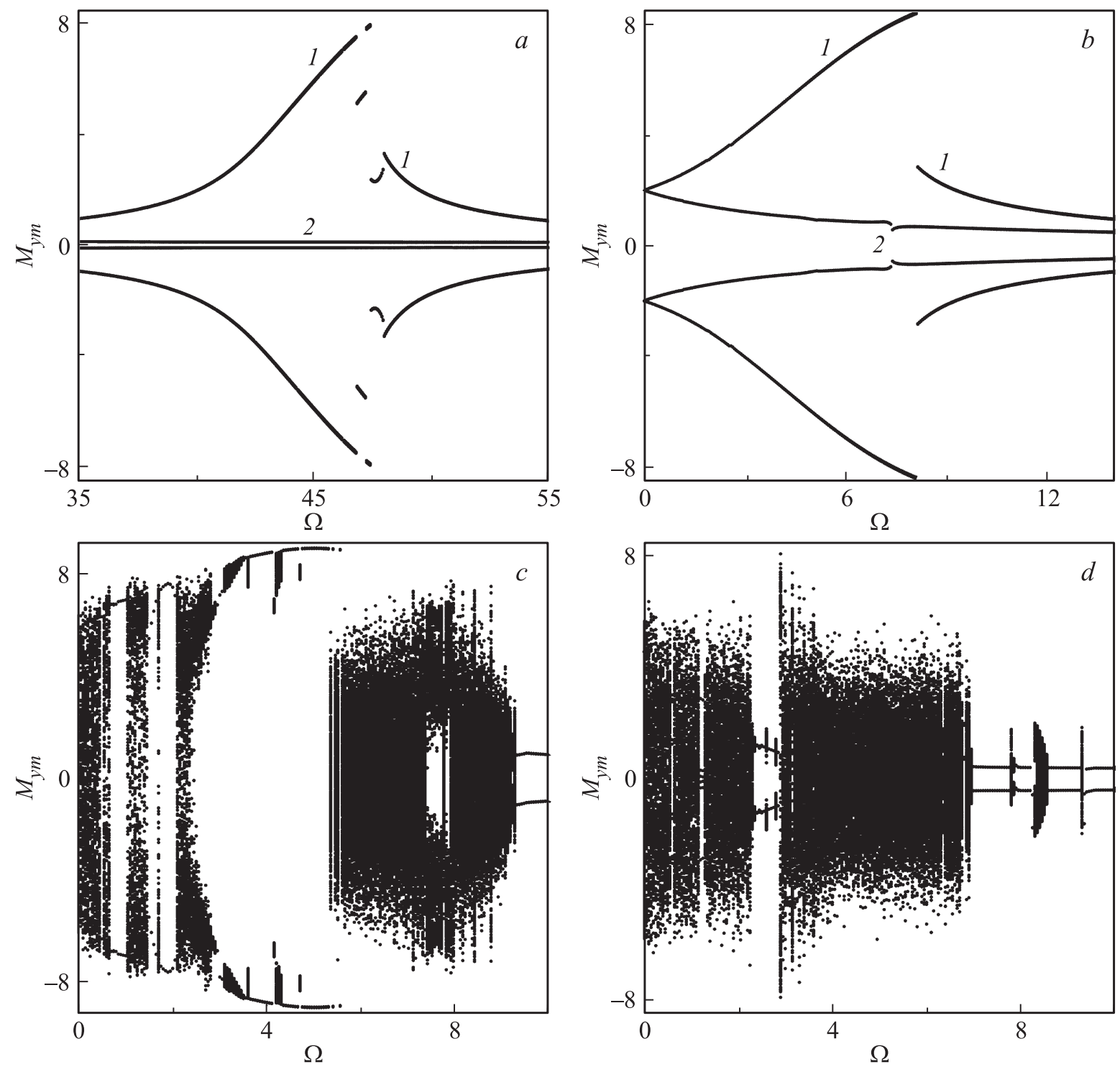

Рис. 6. Диаграммы $\left(M_{y m} ; \Omega\right)$ динамических режимов магнитного момента системы $3 \times 3$ при правой $(1-$ на $a, b ; c)$ и левой $(2-$ на $a, b ; d)$ круговой поляризации переменного поля; амплитуда переменного поля $h_{0}=1$, величина подмагничивающего поля $h_{c}=50,10(a, b)$ и $5(c, d)$.

полностью зачерчивает аттрактор, представляющий собой тор. Рис. 4, с отвечает состоянию бистабильности, т.е. наличию при одних и тех же параметрах двух аттракторов, выбор между которыми реализуется в результате действия различных флуктуаций в динамической системе. В данном случае первым режимом, входящим в бистабильность, является режим регулярных колебаний ( 1 на рис. 4,c), а вторым - метастабильный режим хаотической динамики (2 на рис. $4, c)$. Из второго режима после значительного времени хаотических колебаний магнитный момент может выйти на стационарный гармонический режим.

На рис. 5 приведены БД для динамических режимов при подмагничивающем поле $h_{c}=5(a, b)$ и $50(c)$ и двух амплитудах переменного поля: $h_{0}=0.5(a ; c-$ кривая 1$)$ и 1.5 ( $b ; c-$ кривая 2$)$. Видно, что в случае до- статочно больших подмагничивающих полей (рис. 5,c) увеличение амплитуды переменного поля приводит к сдвигу резонансных частот в область бо́льших значений, а также к росту амплитуды прецессии как при основном резонансе, так и на дополнительных резонансных частотах. В случае же малых подмагничивающих полей наряду с отмеченным происходит расширение частотных интервалов, отвечающих хаотической динамике. Заметим также, что при малых $h_{c}$ имеют место „резонансные“ интервалы частот, в которых амплитуда колебаний магнитного момента практически не изменяется.

В случае круговой поляризации плоскостного переменного поля БД динамических режимов для решеток $3 \times 3$ приведены на рис. 6 при подмагничивающем поле $h_{c}=50,10(a, b)$ и $5(c, d)$ и амплитуде переменного поля $h_{0}=1$. Зависимости 1 на рис. $6, a, b$ и зависимость 

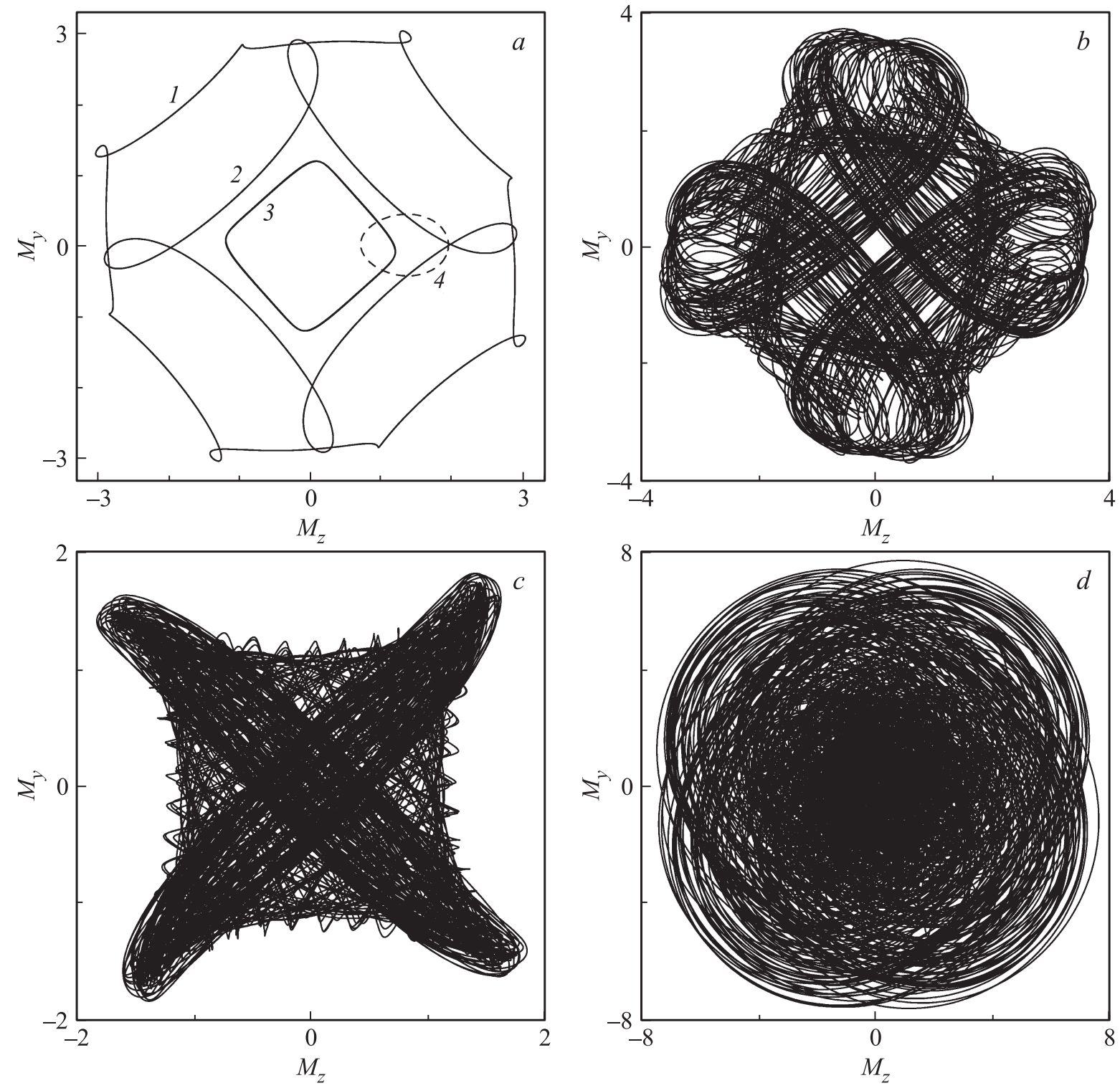

Рис. 7. Проекции на плоскость решетки траекторий регулярных $(a)$ и хаотических $(b-d)$ режимов прецессии магнитного момента системы $3 \times 3$ при левой круговой поляризации переменного поля с $h_{0}=1$ на частотах $\Omega=0.6(1), 1.2(2), 2.5(3), 7(4)(a)$, $1.3(b), 2.6(c)$ и $2.9(d) ; h_{c}=5$.

на рис. 6,c отвечают правой круговой поляризации переменного поля $h_{p y}=h_{0} \cos (\Omega \tau)$ и $h_{p z}=h_{0} \sin (\Omega \tau)$; зависимости 2 на рис. $6, a, b$ и зависимость на рис. $6, d$ отвечают левой круговой поляризации: $h_{p y}=h_{0} \cos (\Omega \tau)$ и $h_{p z}=-h_{0} \sin (\Omega) \tau$. Видно, что в большинстве случаев положение и амплитуда резонанса при правой круговой поляризации переменного поля близки к соответствующим характеристикам при линейной поляризации. При левой круговой поляризации резонанс отсутствует. Только в случае достаточно малых подмагничивающих полей (рис. $6, c, d$ ) на малых частотах устанавливаются высокоамплитудные хаотические колебания $\left(M_{y \max } \approx 6\right)$ и имеют место узкие частотные интервалы, отвечающие регулярным колебаниям с амплитудой $M_{y \max } \approx 3$.
На рис. 7 приведены проекции на плоскость решеток траекторий регулярных $(a)$ и хаотических $(b-d)$ режимов прецессии магнитного момента системы $3 \times 3$ при левой круговой поляризации переменного поля с $h_{0}=1$ на частотах $\Omega=0.6,1.2,2.5,7$ ( $a$, кривые $1-4)$ и 1.3, 2.6, $2.9(b-d)$ при подмагничивающем поле $h_{c}=5$. Видно, что при данной поляризации переменного поля на аттракторах как регулярных, так и хаотических режимов сказывается геометрия (в данном случае квадратная) дипольной решетки: аттракторы имеют ось симметрии четвертого порядка. Исключение составляют регулярные режимы низкоамплитудных колебаний (рис. 7, $a$, кривая 4), которые могут быть различно ориентированы (когда ось прецессии не совпадает с нормалью к системе диполей). 
Отметим, что проведенный анализ может быть обобщен на случай наличия в структуре одноосной анизотропии с осью анизотропии, направленной вдоль нормали к решетке. При этом статическое поле необходимо заменить на $H_{c} \rightarrow H_{c}+\beta m$, где константа анизотропии $\beta$ может быть как положительной (,легкая ось“), так и отрицательной (,легкая плоскость“).

\section{5. Заключение}

Исследование динамических режимов магнитного момента квадратных дипольных решеток в случае лежащего в плоскости системы переменного поля и ориентированного по нормали подмагничивающего поля показало, что в сравнении с единичным диполем частота резонанса оказывается смещенной в область меныших значений. При этом выявляются дополнительные резонансы, в которых амплитуда колебаний может быть сравнима с амплитудой на основной (более низкой) частоте и определяется величинами подмагничивающего и переменного полей, а также размером дипольной решетки. При рассмотрении систем $2 \times 2,3 \times 3$ и $4 \times 4$ наибольшая амплитуда колебаний магнитного момента в дополнительном резонансе выявлена для решеток $3 \times 3$.

Среди динамических режимов имеют место близкие к гармоническим регулярные колебания, при которых переменными являются плоскостные компоненты магнитного момента системы, а также хаотические колебания, возникающие в случае слабых подмагничивающих полей (сравнимых с амплитудой переменного поля). При увеличении числа диполей в решетке частотные интервалы, соответствующие хаотической динамике, расширяются. Обнаружены также квазипериодические режимы и бистабильные состояния, в которые входят регулярный режим и хаотический режим колебания с более низкой амплитудой. Входящий в бистабильность хаотический режим является метастабильным, так как осуществляется самопроизвольный переход от хаотического аттрактора к аттрактору периодических колебаний. В области слабых подмагничивающих полей имеют место интервалы резонансных частот, в которых амплитуда колебаний близка к максимальной (к значению насыщения магнитного момента системы). В случае круговой поляризации переменного поля резонанс возникает только при правой поляризации. При левой круговой поляризации поля в случае слабых подмагничивающих полей устанавливающиеся регулярные и хаотические колебания имеют траектории, отражающие геометрию дипольной решетки.

\section{Список литературы}

[1] R. Skomski. J. Phys.: Condens. Matter 15, R841 (2003).

[2] A.А. Фраерман. УФН 182, 1345 (2012).

[3] П.В. Бондаренко, А.Ю. Галкин, Б.А. Иванов. ЖЭТФ 139, 1127 (2011).
[4] С.А. Дзян, Б.А. Иванов. ЖЭТФ 142, 969 (2012).

[5] С.А. Дзян, Б.А. Иванов. ЖЭТФ 143, 1131 (2013).

[6] Ю.П. Иванов, А.И. Ильин, Е.В. Пустовалов, Л.А. Чеботкеич. ФТТ 52, 1576 (2010).

[7] В.А. Кособукин, Б.Б. Кричевцов. ФТТ 52, 759 (2010).

[8] С.А. Гусев, Ю.Н. Ноздрин, М.В. Сапожников, А.А. Фраерман. УФН 170, 331 (2000).

[9] И.Р. Каретникова, И.М. Нефедов, М.В. Сапожников, А.А. Фраерман, И.А. Шерешевский. ФТТ 43, 2030 (2001).

[10] A.M. Shutyǐ, S.V. Eliseeva, D.I. Sementsov. Phys. Rev. B 91, 024421 (2015).

[11] A.M. Shuty̌́, D.I. Sementsov. J. Magn. Magn. Mater. 401, 1033 (2016).

[12] А.М. Шутый. ЖЭТФ 145, 1048 (2014).

[13] А.М. Шутый, Д.И. Семенцов. Письма в ЖЭТФ 99, 806 (2014).

[14] А.Г. Гуревич, Г.А. Мелков. Магнитные колебания и волны. Наука, М. (1994). 464 с.

[15] А.Ю. Лоскутов, А.С. Михайлов. Основы теории сложных систем. НИЦ „РХД“, Ижевск (2007). 620 с.

[16] Д.И. Семенцов, А.М. Шутый. УФН 177, 831 (2007). 